Bogolyubov Institute for Theoretical Physics, Nat. Acad. of Sci. of Ukraine

(14b, Metrolohichna Str., Kyiv 03680,Ukraine; e-mail: vgrytsay@bitp.kiev.ua)

PACS 05.45.-a, 05.45.Pq 05.65. $+\mathrm{b}$

\title{
SELF-ORGANIZATION AND FRACTALITY IN THE METABOLIC PROCESS OF GLYCOLYSIS
}

\begin{abstract}
Within a mathematical model, the metabolic process of glycolysis is studied. The general scheme of glycolysis is considered as a natural result of the biochemical evolution. By using the theory of dissipative structures, the conditions of self-organization of the given process are sought. The autocatalytic processes resulting in the conservation of cyclicity in the dynamics of the process are determined. The conditions of breaking of the synchronization of the process, increase in the multiplicity of a cyclicity, and appearance of chaotic modes are studied. The phase-parametric diagrams of a cascade of bifurcations, which characterize the transition to chaotic modes according to the Feigenbaum scenario and the intermittence, are constructed. The strange attractors formed as a result of the funnel effect are found. The complete spectra of Lyapunov indices and divergences for the obtained modes are calculated. The values of KS-entropy, horizons of predictability, and Lyapunov dimensions of strange attractors are determined. Some conclusions concerning the structural-functional connections in glycolysis and their influence on the stability of the metabolic process in a cell are presented.

Keywords: glycolysis, metabolic process, self-organization, fractality, strange attractor, Feigenbaum scenario.
\end{abstract}

Glycolysis is one of the oldest systems of biochemical reactions that split hexoses. Possibly, it was formed in protobionts, which were primary cells of the Earth (3.5-4 bln years ago). Those cells were anaerobic heterotrophs. As a nutriment, they used organic substances of the abiogenic origin, which were created by chemo- and phototrophs. Moreover, they got the energy for themselves from the oxidation and the fermentation in Earth's primary oxygenless atmosphere. At the present time, the given biochemical process as a result of the evolution is present practically in all cells, which indicates its relict origin. Namely from glycolysis starts the metabolic process of anaerobic catabolism of glucose, which is completed by the formation of pyruvate. Then the product of glycolysis can be used in three ways: the com-

(C) V.I. GRYTSAY, 2015

ISSN 2071-0194. Ukr. J. Phys. 2015. Vol. 60, No. 12 plete oxidation to $\mathrm{CO}_{2}$ and water under aerobic conditions and the fermentation to lactate or ethanol under anaerobic ones. Glycolysis includes 10 successive reactions, which are running under the action of enzymes in the cytoplasm of cells and are not connected with membrane systems.

But the following questions permanently arise: How was the unique stable sequence of the reactions of glycolysis running in cytoplasm, amorphous at first glance, formed from the huge number of organic substances present in the primary broth and which mechanism of their selection was? In author's opinion, the answers should be sought on the basis of the general theory of chemical evolution by Professor A.P. Rudenko [1-3].

This theory allows one to solve the problems related to the moving forces and the mechanism of evolution in catalytic systems. On this way, the laws of 
the chemical evolution and the selection of elements and structures, as well as their causal dependence, are studied. The complexity of a chemical organization and a hierarchy of chemical systems are a consequence of the evolution. With reagents, catalysts form an intermediate (multiplet) complex possessing the properties of a transient state. Such complex exists in the form of continuously varying configurations on some small section of the reaction path. However, in the course of the catalytic reactions accompanied by a constant inflow of new portions of reagents and an outflow of ready products, the complexes are multiply reproduced. They take the status of elementary open catalytic systems (EOCSs). Rudenko indicated the particular dynamical type of stability of such systems. It can be quantitatively characterized in terms of the intensity of exchange of substances and the energy of the basic reaction, which is equal to the product of the activity of a catalytic center by the elementary chemical affinity of the basic reaction.

Thus, Rudenko made conclusion that there occurs the natural selection of the catalytic centers with the highest activity in the process of self-development of EOCSs. On those centers, the basic reaction is concentrated more and more. The centers with lower activity are gradually eliminated from the kinetic continuum and "do not survive". At the multiple successive changes in EOCSs, the transition to a higher level of steadiness is accompanied by the evolution of the mechanism of the basic reaction due to changes in the composition and the structure of a catalyst operating at the beginning of the reaction, as well as due to the division of the chamical process into elementary stages and the appearance of new catalysts of these stages due to changes in EOCSs.

The above consideration allows us to make the following conclusion. The validity of Rudenko's theory of chemical evolution of elementary open catalytic systems is supported by its correspondence with the general theory of dissipative structures [4]. Just the dissipative structures arising in open nonlinear systems of the Nature are the reason for the selforganization in it. At the appearance of an autocatalytic oscillatory process in such dissipative system, the system becomes self-developing. Those paths of evolutionary changes are formed with the highest rate and the probability, on which the absolute catalytic activity increases maximally.
During the chemical evolution on the Earth at the appearance of favorable conditions for the creation of organic substances, the enzymatic reactions replaced rapidly and completely the inorganic catalysis. In the primary broth, the almost infinite number of open biochemical systems were formed. But only those of them were evolved, in which the autocatalysis was most intense. As a result of the selforganization of the given elementary biochemical processes, the metabolic networks with the ability to a self-assembling were formed in larger interconnected open nonlinear systems. This occurred until a cell was created. One of the biochemical processes conserved from the previous metabolism is glycolysis.

A lot of experimental and theoretical works are devoted to the study of glycolysis. The researchers were expecially interested in the glycolytic oscillations observed in yeast cells [5]. The experimental characteristics were obtained, and a number of mathematical models of this process were constructed [6]. Sel'kov proposed a fine theoretical model [7], in which the enzyme phosphofructokinase is activated by its. Then Goldbeter and Lefever developed a more detailed model [8], in which the allosteric nature of phosphofructokinase was taken into account.

The other mathematical models of biochemical processes can be found, for example, in [9-14]. The obtained results enrich our knowledge about the given processes.

The study presented in this work will be based on the mathematical model of glycolysis and gluconeogenesis constructed by Professor V.P. Gachok [15-17]. A specific feature of his model consists in the consideration of the action of such factors as the adeninenucleotide cycle and the feedback of gluconeogenesis on the allosteric enzyme phosphofructokinase. This allowed him to analyze more qualitatively the reasons for the appearance of an oscillatory dynamics in glycolysis.

In what follows, the model is improved so that the complete chain of the whole metabolic process of glycolysis from start to end under anaerobic conditions is modeled. This enables us to study the positive feedbacks of the process, which create a stable autocatalytic process of the given section of metabolism irrespective of other metabolic processes in a cell. Glycolysis is considered as an open part of the biosystem, which is self-organized by itself due to

ISSN 2071-0194. Ukr. J. Phys. 2015. Vol. 60, No. 12 


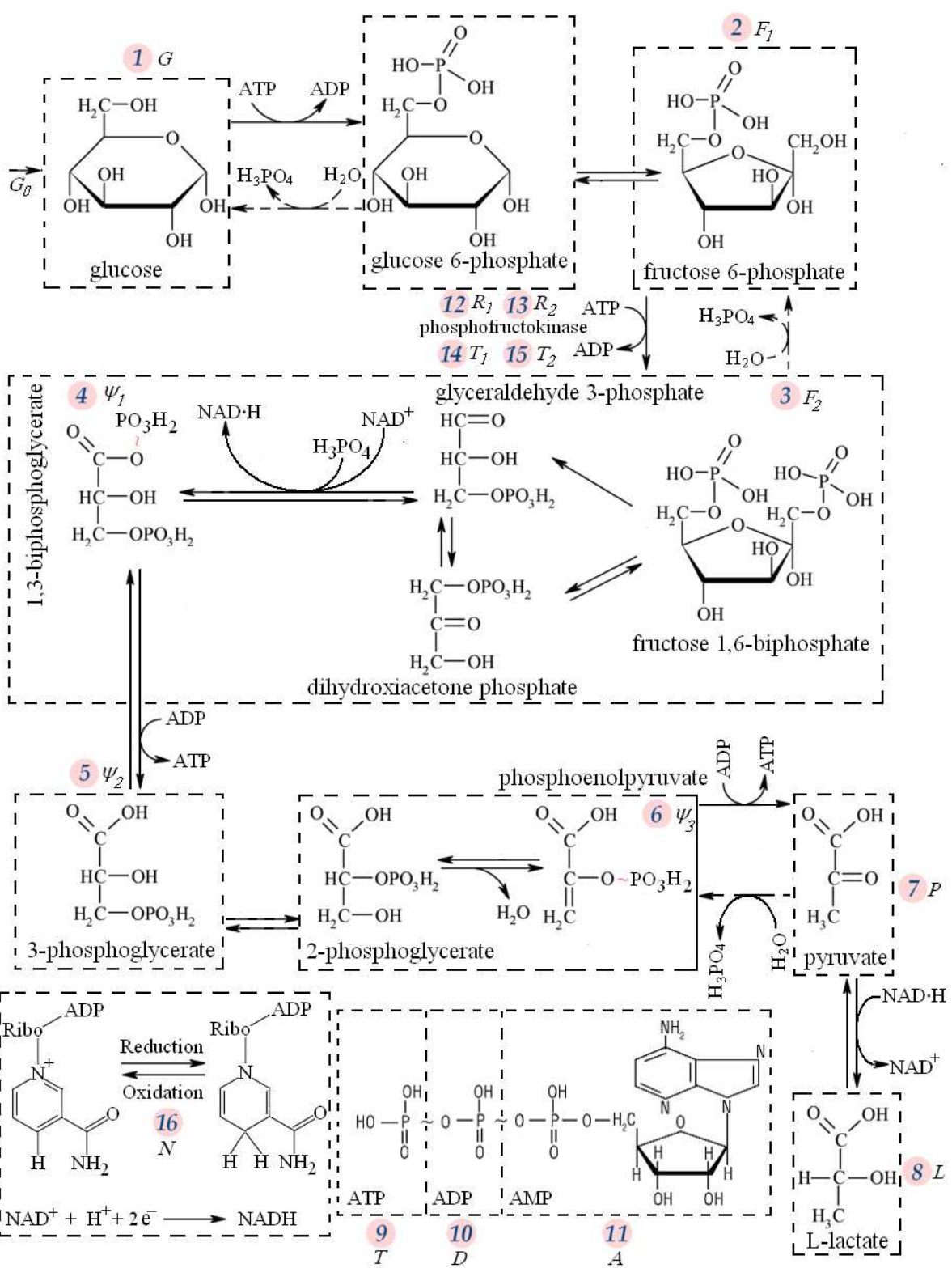

Fig. 1. General scheme of the metabolic process of glycolysis

the input substances and output products of the reaction in a cell, ensuring the condition of its survival and evolution.

\section{Mathematical Model}

The general scheme of the process of glycolysis is presented in Fig. 1. According to it, the mathematical model (1)-(16) is constructed with regard for the mass balance and the enzymatic kinetics.
Here, we will describe the process of catabolism of glucose to lactate under anaerobic conditions. The insignificant changes in Eq. (8) and the replacement of the enzyme lactate dehydrogenase by pyruvate decarboxylase allow the application of this model to the study of the process of alcoholic fermentation. In this case, the model will describe the formation of ethanol instead of lactate. The internal dynamics of process given by the solution of Eq. (1)-(16) remains 
invariable.

$\frac{d G}{d t}=\frac{G_{0}}{S} \frac{m_{1}}{m_{1}+F_{1}}-l_{8} V(G) V(T)$,

$\frac{d F_{1}}{d t}=l_{8} V(G) V(T)-l_{1} V\left(R_{1}\right) V\left(F_{1}\right) V(T)+$

$+l_{5} \frac{1}{1+\gamma A} V\left(F_{2}\right)-m_{3} \frac{F_{1}}{S}$,

$\frac{d F_{2}}{d t}=l_{1} V\left(R_{1}\right) V\left(F_{1}\right) V(T)-l_{5} \frac{1}{1+\gamma A} V\left(F_{2}\right)-m_{5} \frac{F_{2}}{S}$,

$\frac{d \psi_{1}}{d t}=\frac{m_{5}\left(F_{2} / S\right)}{S_{1}+m_{5}\left(F_{2} / S\right)}-l_{6} V\left(\psi_{1}\right) V(D)+$

$+m_{7} V(M-N) V(P)$,

$\frac{d \psi_{2}}{d t}=l_{6} V\left(\psi_{1}\right) V(D)-m_{8} \frac{\psi_{2}}{S}$,

$\frac{d \psi_{3}}{d t}=\frac{\psi_{2}}{S} \frac{m_{2}}{m_{2}+\psi_{3}}-l_{2} V\left(\psi_{3}\right) V(D)-m_{4} \frac{\psi_{3}}{S}$,

$\frac{d P}{d t}=l_{2} V\left(\psi_{3}\right) V(D)-m_{6} \frac{P}{S}-l_{7} V(N) V(P)$,

$\frac{d L}{d t}=l_{7} V(N) V(P)-m_{9} \frac{L}{S}$,

$\frac{d T}{d t}=l_{2} V\left(\psi_{3}\right) V(D)-l_{1} V\left(R_{1}\right) V\left(F_{1}\right) V(T)+$

$+l_{3} \frac{A}{\delta+A} V(T)-l_{4} \frac{T^{4}}{\beta+T^{4}}+$

$+l_{6} V\left(\psi_{1}\right) V(D)-l_{9} V(G) V(T)$,

$\frac{d D}{d t}=l_{1} V\left(R_{1}\right) V\left(F_{1}\right) V(T)-l_{2} V\left(\psi_{3}\right) V(D)+$

$+2^{*} l_{3} \frac{A}{\delta+A} V(T)-l_{6} V\left(\psi_{1}\right) V(D)+$

$+l_{9} V(G) V(T)$,

$\frac{d A}{d t}=l_{4} \frac{T^{4}}{\beta+T^{4}}-l_{3} \frac{A}{\delta+A} V(T)$,

$\frac{d R_{1}}{d t}=k_{1} T_{1} V\left(F_{1}^{2}\right)+k_{3} R_{2} V\left(D^{2}\right)-$

$-k_{5} R_{1} \frac{T}{1+T+\alpha A}-k_{7} R_{1} V\left(T^{2}\right)$,

$\frac{d R_{2}}{d t}=k_{5} R_{1} \frac{T}{1+T+\alpha A}-k_{3} R_{2} V\left(D^{2}\right)+$

$+k_{2} T_{2} V\left(F_{1}^{2}\right)-k_{8} R_{2} V\left(T^{2}\right)$,

$$
\begin{aligned}
& \frac{d T_{1}}{d t}=k_{7} R_{1} V\left(T^{2}\right)-k_{6} T_{1} \frac{T}{1+T+\alpha A}+ \\
& +k_{4} T_{2} V\left(D^{2}\right)-k_{1} T_{1} V\left(F_{1}^{2}\right), \\
& \frac{d T_{2}}{d t}=k_{6} T_{1} \frac{T}{1+T+\alpha A}-k_{4} T_{2} V\left(D^{2}\right)- \\
& -k_{2} T_{2} V\left(F_{1}^{2}\right)+k_{8} R_{2} V\left(T^{2}\right), \\
& \frac{d N}{d t}=-l_{7} V(N) V(P)+l_{7} V(M-N) V\left(\psi_{1}\right) .
\end{aligned}
$$

Here, $V(X)=X /(1+X)$ is the function accounting for the adsorption of the enzyme in the region of local coupling. The variables of the system of equations are made dimensionless [15-17].

We take the following values of parameters:

$l_{1}=0.0535 ; \quad l_{2}=0.046 ; \quad l_{3}=0.0017 ;$

$l_{4}=0.01334 ; \quad l_{5}=0.3 ; \quad l_{6}=0.001 ; \quad l_{7}=0.01 ;$

$l_{8}=0.0535 ; \quad l_{9}=0.001 ; \quad k_{1}=0.07 ; \quad k_{2}=0.01 ;$

$k_{3}=0.0015 ; \quad k_{4}=0.0005 ; \quad k_{5}=0.05 ;$

$k_{6}=0.005 ; \quad k_{7}=0.03 ; \quad k_{8}=0.005 ; \quad m_{1}=0.3 ;$

$m_{2}=0.15 ; \quad m_{3}=1.6 ; \quad m_{4}=0.0005 ; \quad m_{5}=0.007$;

$m_{6}=10 ; \quad m_{7}=0.0001 ; \quad m_{8}=0.0000171$;

$m_{9}=0.5 ; \quad G_{0}=18.4 ; \quad L=0.005 ; \quad S=1000 ;$

$A=0.6779 ; \quad M=0.005 ; \quad S_{1}=150 ; \quad \alpha=184.5 ;$

$\beta=250 ; \quad \delta=0.3 ; \quad \gamma=79.7$.

The mathematical model is given by the system of nonlinear differential equations. The equations correspond to basic sections of the metabolic process, which define the sequence of reactions and affect the stability of glycolysis. Some parts of the metabolic network, which affect insignificantly the self-organization of the process, are described generically. In Fig. 1, we show the parts of the metabolic network (from 1 to 16) corresponding to the number of a differential equation: (1)-(16).

On the first stage (1), the incoming substance $G_{0}$ (glucose) is phosphorylated with the help of the enzyme hexokinase to glucose-6-phosphate. The donor of a phosphorylic group is a molecule ATP $(T)(1)$, (9). This reaction is running irreversibly. The molecules of glucose-6-phosphate cannot leave a cell. In this

ISSN 2071-0194. Ukr. J. Phys. 2015. Vol. 60, No. 12 
case, they are a product of the reaction and an allosteric inhibitor. When the concentration of glucose6-phosphate in the cell exceeds the normal level, glucose-6-phosphate inhibits temporarily and reversibly hexokinase $(1)\left(F_{1}\right)$, so that the rate of its formation is put in correspondence with the rate of its consumption in the subsequent reaction. Then, with the participation of the enzymes phosphohexoisomerase (or phosphoglucoseisomerase), the reversible isomerization of glucose-6-phosphate in fructose-6-phosphate, which does not influence the irreversibility of the process, occurs.

Equations (2), (3) describe the process of formation of fructose-6-phosphate $\left(F_{1}\right)$ and its transformation into fructose-1,6-biphosphate $\left(F_{2}\right)$. This happens under the catalytic action of the key enzyme of glycolysis - phosphofructokinase. This enzyme catalyzes the irreversible transfer of a phosphorylic group from ATP (2), (9) to fructose-6-phosphate with the formation of fructose-1,6-biphosphate. The substrate fructose-6-phosphate is an activator, whereas ATP is an inhibitor of the given process. In addition to such regulation, the given enzyme can be regulated by the adeninenucleotidic cycle: ATPADP-AMP (see below), which favors the support of an optimal stable stationary state. We now describe the process of gluconeogenesis. At low concentrations of reagents, the reaction is reversible $F_{2} \rightarrow F_{1}(2),(3)$, and the positive feedback affecting the stability of the process is created. The subsequent splitting of fructose-1,6-biphosphate into two different triosephosphates (glyceraldehyde-3-phosphate and dihydroxyacetonephosphate) occurs reversibly.

Equation (4) describes the formation of 1,3-biphosphoglycerate $\left(\psi_{1}\right)$, which is the start of the second stage of glycolysis. With the help of the enzyme $D$-glyceraldehydephosphate dehydrogenase, glyceraldehyde-3-phosphate is oxidized and joins phosphoric acid. In this case, the role of an acceptor of hydrogen is played by coenzyme $\mathrm{NAD}^{+}$. There occurs the enzymatic reduction: $\mathrm{NAD}^{+} \rightarrow \mathrm{NAD} \cdot \mathrm{H}$ (4), (16).

With the help of Eq. (5), we describe the process of transfer of a high-energy phosphorylic group by the enzyme phosphoglycerate kinase from a carboxyl of 1,3-biphosphoglycerate onto ADP. As a result, ATP (11) and 3-phosphoglycerate $\psi_{2}$ are formed.

Equation (6) describes the formation of 2-phosphoglycerate with the help of the enzyme phosphoglyce- rate mutase. Then water is eliminated, which results in the formation of phosphoenolpyruvate $\psi_{3}$.

The formation of pyruvate $P$ under the action of the enzyme pyruvate kinase is presented by Eq. (7). Here, the phosphorylation of the substrate occurs. As distinct from reactions (4)-(5), where the reverse reaction of gluconeogenesis at the synthetic absorption of $\mathrm{CO}_{2}$ is possible, we observe the powerful irreversible process: $\mathrm{ADP} \rightarrow \mathrm{ATP}$.

Equation (8) characterizes the formation of the second product of the process - lactate $L$. With the help of the enzyme lactate dehydrogenase, there occurs the enzymatic oxidation $\mathrm{NAD} \cdot \mathrm{H} \rightarrow \mathrm{NAD}^{+}$. The balance between $\mathrm{NAD}^{+}$and NAD $\cdot \mathrm{H}$ holds (16).

Equations (9)-(11) describe the kinetics of changes in the contents of $\operatorname{ATP}(9), \operatorname{ADP}(10)$, and AMP (11) according to the metabolic scheme of glycolysis (see above). On the whole, the adeninenucleotidic cycle of mutual transitions between the given reagents arises: ATP-ADP-AMP. This cycle favors the conservation of the optimum stationary state of the metabolic process.

Equations (12)-(15) show the kinetics of changes in the content of the allosteric enzyme phosphofructokinase. It is assumed that the enzyme has two active forms $\left(R_{1}(12)\right.$ and $\left.R_{2}(13)\right)$ and two inactive ones $\left(T_{1}\right.$ (14) and $\left.T_{2}(15)\right)$. In this case, we observe the mutual transformation of forms $T_{1}$ and $R_{1}, T_{2}$ and $R_{2}$. The equations present the general scheme of regulating connections. Form $R_{1}(12)$ is created from form $T_{1}$ as a result of the saturation of two allosteric centers by molecules $F_{1}$ and from form $R_{2}$ at the expense of two molecules $D$. The inactivation of form $R_{1}$ occurs at the expense of $T(12)$ with the formation of form $R_{2}$ (13) and two molecules $T(12)$ with the formation of form $T_{2}$ (15). This invertible inactivation is inhibited with increase in $A$ according to the high level of $T$ (parameter $\alpha$ ) (12). Equations (13)-(15) are constructed analogously.

Equation (16) describes the kinetics of changes in the content of the reduced form nicotineamideadeninenucleotide $\mathrm{NAD} \cdot \mathrm{H}$, according to its consumption and the reduction of oxidized form $\mathrm{NAD}^{+}(4)$. The balance between the oxidized and reduced forms in the glycolytic cycle is conserved in the invariable form. In this case, the integral of motion NAD $\cdot \mathrm{H}(t)+$ $+\mathrm{NAD}^{+}(t)=M$ is satisfied.

The study of solutions of the present mathematical model (1)-(16) is realized with the help of the 

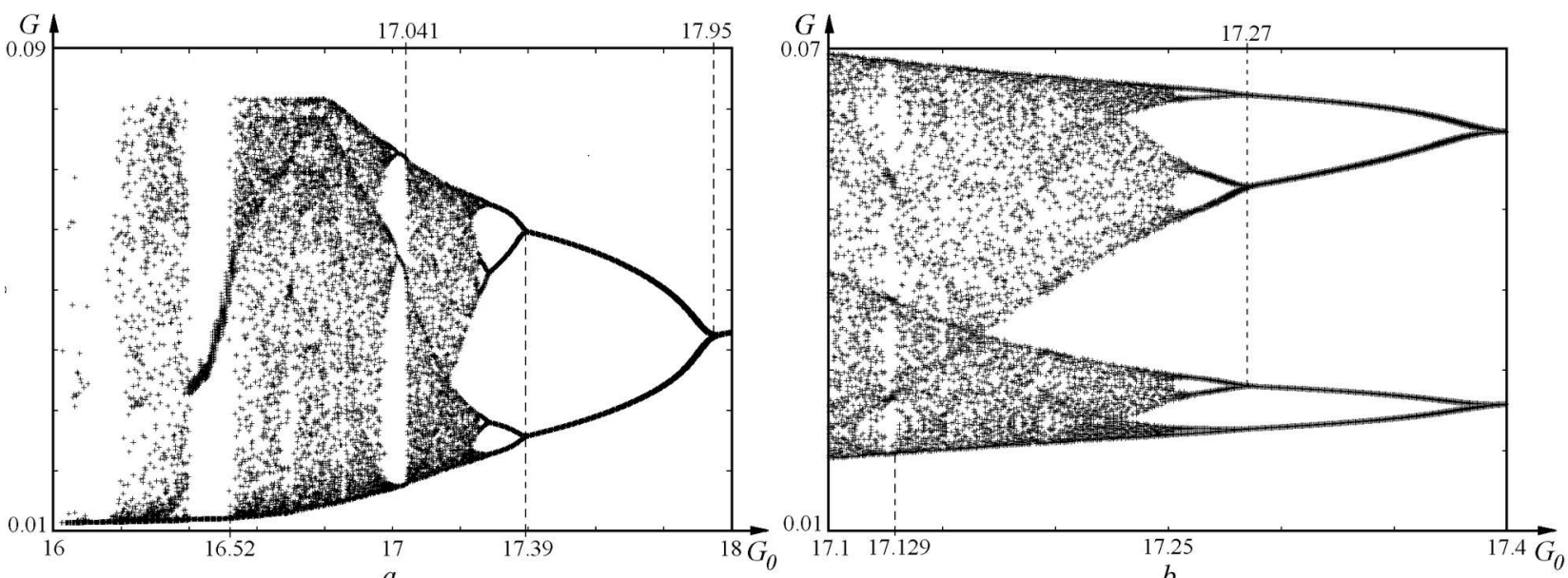

Fig. 2. Phase-parametric diagram of the system for the variable $G(t): a-G_{0} \in(16,18) ; b-G_{0} \in(17.1,17.4)$

theory of nonlinear differential equations $[18,19]$ and the methods of modeling of biochemical systems used earlier in works [20-40].

The numerical solution of this autonomous system of nonlinear differential equations was made within the Runge-Kutta-Merson method. The accuracy of calculations was set to be $10^{-8}$. For the reliability of studies, namely for the system being on the transient initial state to approach the asymptotic solution with an attractor, the duration of calculations was taken to be $10^{6}$. For this time, the trajectory "sticks" the appropriate attractor.

\section{Results of Studies}

The given mathematical model is a system of nonlinear differential equations (1)-(16), which describes the open nonlinear biochemical system. For it, the input substance is glucose characterized by the coefficient $G_{0}$. The output products of the reaction are lactose, ATP, and $\mathrm{H}_{2} \mathrm{O}$. Namely the flows of these substances form the internal dynamics of the given metabolic process. At the breaking of the mass balance between them, the continuity of the running of glycolysis is violated as well. From the energetic viewpoint, the transformation of glucose in pyruvate means a significant decrease in the free energy of the products of the reaction. Therefore, glycolysis is the energy-gained irreversible process running in the open nonlinear system far from equilibrium. In addition, the whole metabolic process of glycolysis is embraced by a feedback formed by the redox reac-

1256 tions of transfer of electrons with the help of NAD (16) (Fig. 1). Due to NAD, glycolysis includes the autocatalytic process of catabolism of glucose. After the successive splitting of a molecule of glucose and the appearance of 2 molecules (ATP and a molecule of pyruvate) on the output, the system returns in the initial state. Thus, the metabolic process of glycolysis in a cell can be separated as a united self-regulating complex. The whole metabolic process of glycolysis can be considered as the process of self-organization, which is functioning in the cyclic mode. When the evolution of metabolic processes in protobionts was completed, glycolysis remains to be invariable in all cells.

Let us study the dependence of the oscillatory dynamics of the metabolic process of glycolysis on $G_{0}$. Figure 2, $a, b$ presents the constructed phaseparametric diagrams of the system for the variable $G(t)$, when $G_{0}$ changes in the corresponding intervals. To construct the phase-parametric diagrams, we used the method of cuts. In the phase space containing the trajectories of the system, we place a cutting plane at $R_{1}=0.7$. If the trajectory crosses this plane in a certain direction, the value of the chosen variable (in this case, $G(t)$ ) is placed on the phase-parametric diagram. Such choice is explained by the symmetry of oscillations of the active form of the allosteric enzyme phosphofructokinase relative to this point in many modes calculated earlier. For every given value of $G_{0}$, we mark the intersection of this plane in a single direction by the trajectory, when the trajectory has approached the attractor. In the case where a multiple

ISSN 2071-0194. Ukr. J. Phys. 2015. Vol. 60, No. 12 

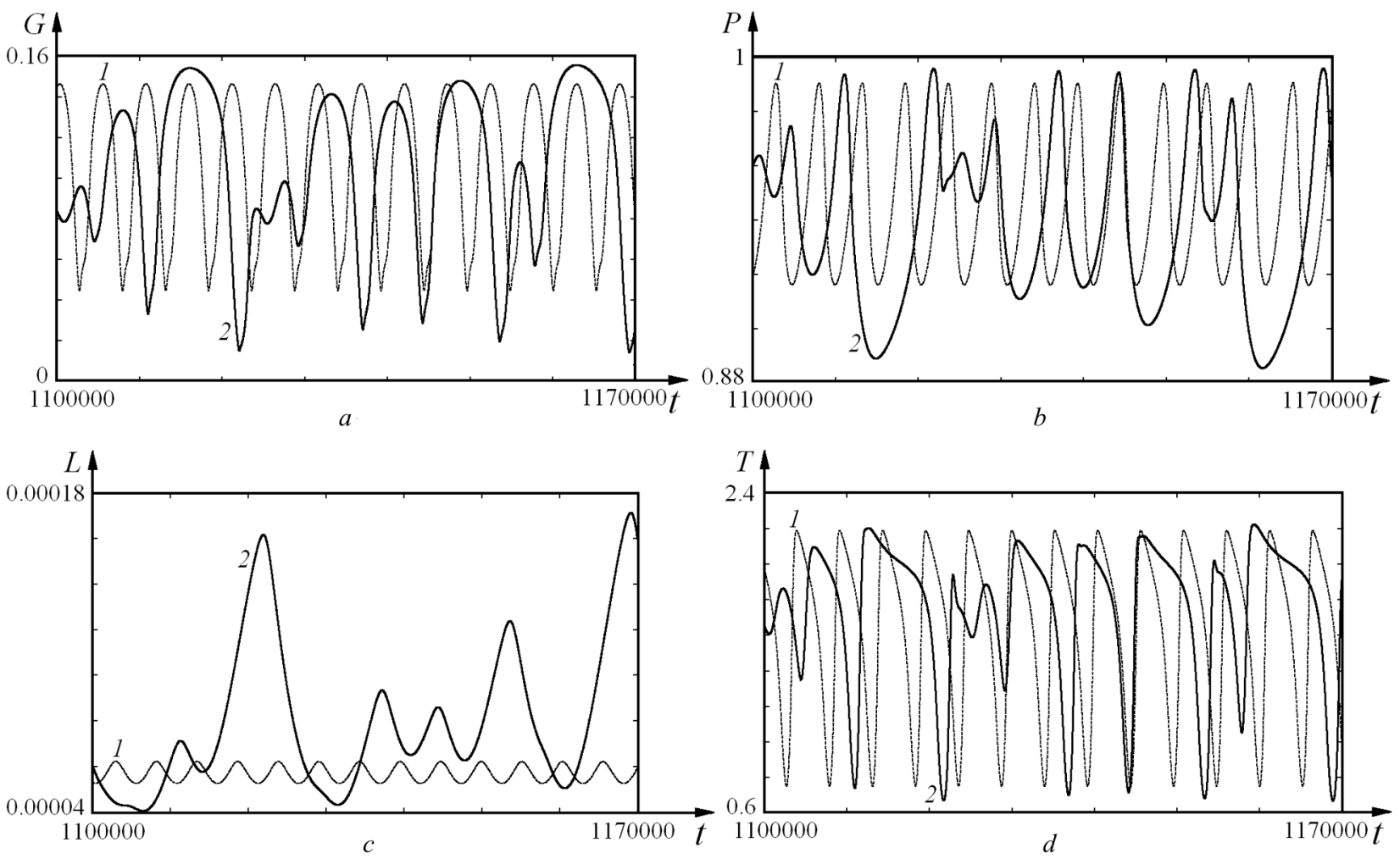

Fig. 3. Kinetic curves for the variables: $G(t)(a), P(t)(b), L(t)(c)$, and $T(t)(d)$ in the 1-fold periodic mode for $G_{0}=19(1)$ and in the chaotic mode for $G_{0}=16.7$ (2)

periodic limiting cycle arises, we mark a number of points on the plane. These points coincide in the period. If a deterministic chaos arises, then such points, where the trajectory intersects the plane, are positioned chaotically.

Consider the diagrams from right to left. It is seen from the phase-parametric diagrams that, for $G_{0}^{j}=17.95$, the period of oscillations is doubled. For $G_{0}^{j+1}=17.39$, we observe the repeated doubling of the period. Then, for $G_{0}^{j+2}=17.27$, the period of autooscillations is doubled once more. As $G_{0}$ decreases further, no doubling of the period of autooscillations occurs, and a chaotic mode arises as a result of the intermittence. The determined sequence of bifurcations satisfies the relation

$\lim _{t \rightarrow \infty} \frac{G_{0}^{j+1}-G_{0}^{j}}{G_{0}^{j+2}-G_{0}^{j+1}} \approx 4.667$.

This number is very close to the universal Feigenbaum constant. Thus, as the parameter $G_{0}$ decreases on this section, the doubling of the period of autooscillations occurs according to the Feigenbaum scenario
[41]. This means that, in the given unstable modes of the physical system, any arising fluctuation can induce a chaotic cyclic mode.

For $G_{0}=17.129$ (Fig. 2, b) and $G_{0}=17.041$, $G_{0}=16.52$ (Fig. 2, a), the windows of periodicity arise. The deterministic chaos is destroyed, and periodic and quasiperiodic modes are established. Outside these windows, chaotic modes arise. The identical windows of periodicity are observed also on less scales of the diagram. In other words, the phaseparametric diagrams on small and large scales are analogous. This indicates the fractal nature of the obtained cascade of bifurcations in the metabolic process of glycolysis.

In Fig. $3, a-d$, we show, as an example, the kinetics of autooscillations for some components of the metabolic process in the 1 -fold periodic mode for $G_{0}=19$ and in the chaotic mode for $G_{0}=16.7$. The synchronous autooscillations of glucose, pyruvate, lactate, and $A T P$ become chaotic.

According to the kinetics, the phase portraits of the system are changed as well. In Fig. $4, a-d$, we present, 

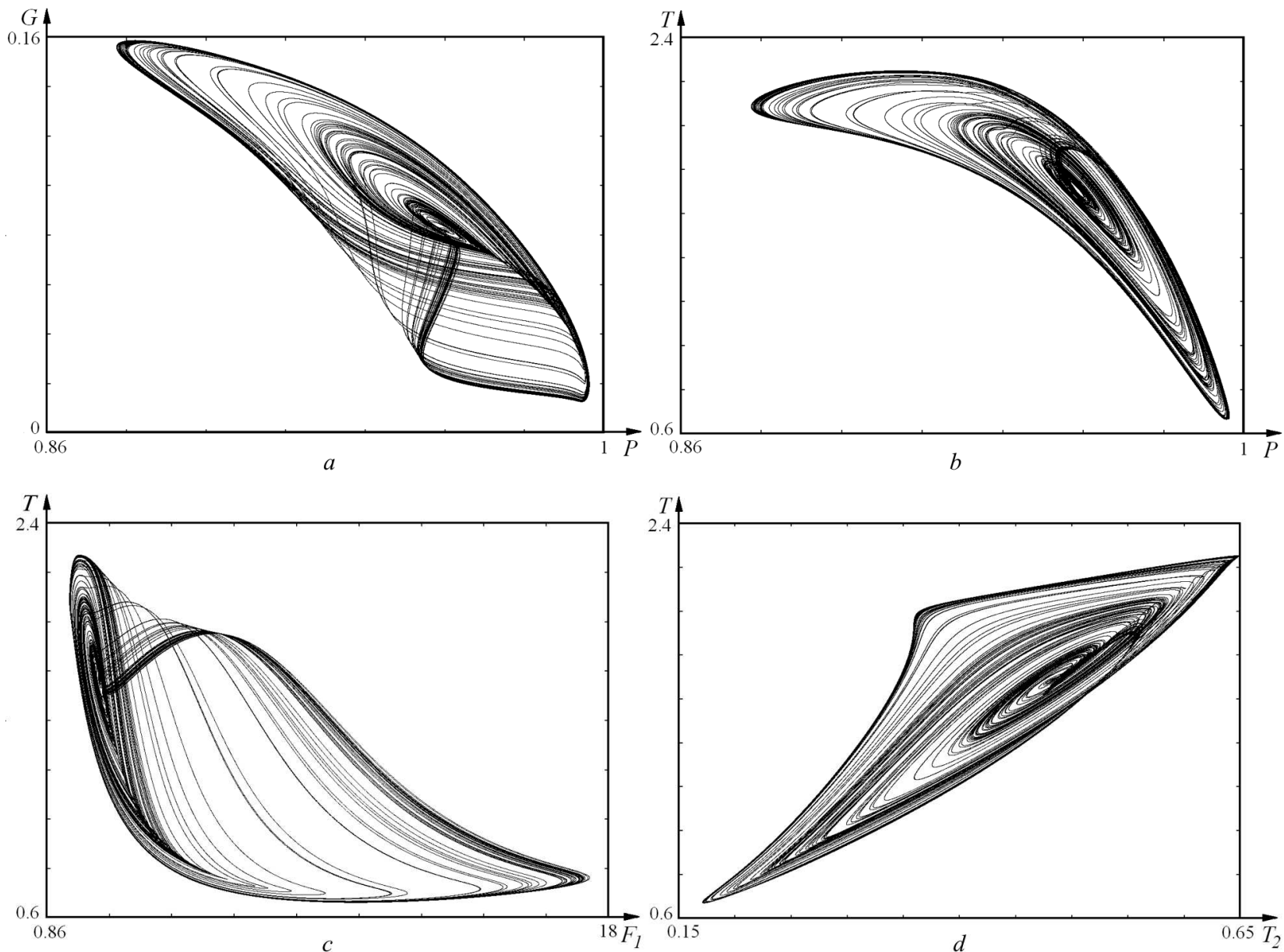

Fig. 4. Projections of the phase portrait of a strange attractor formed for $G_{0}=16.7$ in the appropriate plane: $(P, G) a,(P, T)$ $b,\left(F_{1}, T\right) c$, and $\left(T_{2}, T\right) d$

as an example, some projections of phase portraits of the system for $G_{0}=16.7$. The given chaotic mode is a strange attractor formed as a result of the funnel effect. Inside the formed funnel, we observe the mixing of dispersing trajectories approaching one another. Due to fluctuations, the stability of the cycle is violated, and the cycle becomes chaotic. A deterministic chaos is formed.

While studying the phase-parametric diagrams in Fig. 2, $a, b$, it is impossible beforehand to determine, at which values of the parameter $G_{0}$ a multiple stable (quasistable) autoperiodic cycle or a strange attractor is formed.

For the unique identification of the type of obtained attractors and for the determination of their stability, we calculated the complete spectra of Lya- punov indices and their sums $\Lambda=\sum_{j=1}^{16} \lambda_{j}$ at some chosen points. The calculation was carried out by Benettin's algorithm with the orthogonalization of the perturbation vectors by the Gram-Schmidt method [19].

A specific feature of the calculation of those indicators consists in the complexity of the determination of the perturbation vectors represented by $16 \times 16$ matrices on a personal computer.

The algorithm of calculation of the complete spectrum of Lyapunov indices is as follows. First, some point on the attractor $\overline{X_{0}}$ is taken as the input one. Then the trajectory going out from the point and the evolution of $N$ perturbation vectors are traced. In our case, $N=16$ is the number of variables of the system. The input equations of the system, which are 
supplemented by 16 complexes of equations in variations, are solved numerically. As the initial perturbation vectors, we set the collection of vectors $\overline{b_{1}^{0}}, \overline{b_{2}^{0}}, \ldots$, $\overline{b_{16}^{0}}$, which are orthogonal to one another and are normalized to 1 . In some time $T$, the trajectory comes to the point $\overline{X_{1}}$, and the perturbation vectors are $\overline{b_{1}^{1}}$, $\overline{b_{2}^{1}}, \ldots, \overline{b_{16}^{1}}$. Their renormalization and orthogonalization by the Gram-Schmidt method are realized by the following scheme:

$$
\begin{aligned}
& \overline{b_{1}^{1}}=\frac{\overline{b_{1}}}{\left\|\overline{b_{1}}\right\|}, \\
& \overline{b_{2}^{\prime}}=\overline{b_{2}^{0}}-\left(\overline{b_{2}^{0}}, \overline{b_{1}^{1}}\right) \overline{b_{1}^{1}}, \quad \overline{b_{2}^{1}}=\frac{\overline{b_{2}^{\prime}}}{\left\|\overline{b_{2}^{\prime}}\right\|}, \\
& \overline{b_{3}^{\prime}}=\overline{b_{3}^{0}}-\left(\overline{b_{3}^{0}}, \overline{b_{1}^{1}}\right) \overline{b_{1}^{1}}-\left(\overline{b_{3}^{0}}, \overline{b_{2}^{1}}\right) \overline{b_{2}^{1}}, \quad \overline{b_{3}^{1}}=\frac{\overline{b_{3}^{\prime}}}{\left\|b_{3}^{\prime}\right\|}, \\
& \overline{b_{4}^{\prime}}=\overline{b_{4}^{0}}-\left(\overline{b_{4}^{0}}, \overline{b_{1}^{1}}\right) \overline{b_{1}^{1}}-\left(\overline{b_{4}^{0}}, \overline{b_{2}^{1}}\right) \overline{b_{2}^{1}}-\left(\overline{b_{4}}, \overline{b_{3}^{1}}\right) b_{3}^{1}, \quad \overline{b_{4}^{1}}=\frac{\overline{b_{4}^{\prime}}}{\left\|b_{4}^{\prime}\right\|},
\end{aligned}
$$

$\overline{b_{16}^{\prime}}=\overline{b_{16}^{0}}-\left(\overline{b_{16}^{0}}, \overline{b_{1}^{1}}\right) \overline{b_{1}^{1}}-\left(\overline{b_{16}^{0}}, \overline{b_{2}^{1}}\right) \overline{b_{2}^{1}}-\left(\overline{b_{16}}, \overline{b_{3}^{1}}\right) b_{3}^{1}-\ldots-$ $-\left(\overline{b_{16}}, \overline{b_{15}^{1}}\right) b_{15}^{1}, \quad \overline{b_{16}^{1}}=\frac{\overline{b_{16}^{\prime}}}{\left\|b_{16}^{\prime}\right\|}$,

Then the calculation is continued, by starting from the point $\overline{X_{1}}$ and the perturbation vectors $\overline{b_{1}^{1}}, \overline{b_{2}^{1}}, \ldots$, $\overline{b_{16}^{1}}$. In the next time interval $T$, a new collection of perturbation vectors $\overline{b_{1}^{2}}, \overline{b_{2}^{2}}, \ldots, \overline{b_{16}^{2}}$ is formed. It is again orthogonalized and renormalized by the above scheme. This sequence of operations is repeated a sufficiently large number of times M. In this case, in the course of calculations, we determine the sums

$$
\begin{aligned}
& S_{1}=\sum_{i=1}^{M} \ln \left\|b_{1}^{\prime i}\right\|, \quad S_{2}=\sum_{i=1}^{M} \ln \left\|b_{2}^{\prime i}\right\|, \ldots, \\
& S_{16}=\sum_{i=1}^{M} \ln \left\|b_{16}^{\prime i}\right\|,
\end{aligned}
$$

in which the perturbation vectors before the renormalization, but after the orthogonalization are present.

We evaluate 16 Lyapunov indices in the following way:

$\lambda_{j}=\frac{Z_{j}}{M T}, \quad j=1,2, \ldots, 16$.

ISSN 2071-0194. Ukr. J. Phys. 2015. Vol. 60, No. 12
For comparison, we give the spectra of Lyapunov indices for some modes of the system. For brevity without any loss of information, the values of indices are rounded to the 5 -th decimal point.

The criterion of validity of the calculation is the ratio of Lyapunov indices: $\lambda_{1}>\lambda_{2}>\lambda_{3}>\ldots>\lambda_{16}$. For a regular attractor, it must be: $\lambda_{1} \approx 0$. The next subsequent indices can be also $\approx 0$ in some cases. In other cases, they are negative. The zero value of first Lyapunov index indicate the presence of a stable limiting cycle.

For a strange attractor, the presence of at least one positive Lyapunov index is obligatory. Then the zero index is positioned; the rest indices are negative. The presence of negative indices means the compression of the phase space in the corresponding directions, whereas the positive indices indicate the divergence of trajectories in some directions. Therefore, there occurs the mixing of trajectories in narrow places of the phase space, and the deterministic chaos arises. The Lyapunov indices include obligatorily the zero index. This means the conservation of an aperiodic trajectory of the attractor in some domain of the phase space, which is the condition of existence of a strange attractor.

As an example for comparison, we now give several calculations of the complete spectrum of Lyapunov indices.

For $G_{0}=17.25$, the regular attractor $1 \cdot 2^{2}$ of a quasistable autoperiodic cycle arises.

$\lambda_{1}-\lambda_{16}$ are equal to: $.00000,-.00004,-.00006$, $-.00008,-.00010,-.00054,-.00085,-.00129,-.00484$, $-.00562,-.00562,-.00562,-.00980,-.01002,-.01582$, $-.02343 . \Lambda=-.08373$.

For $G_{0}=17.2$, the strange attractor $1 \cdot 2^{x}$ arises. $\lambda_{1}-\lambda_{16}$ are equal to: .00004, .00000, -.00006, $-.00009,-.00013,-.00053,-.00085,-.00126,-.00483$,

$-.00556,-.00556,-.00556,-.00972,-.00997,-.01588$,

$-.02329 . \Lambda=-.08325$.

For $G_{0}=16.8$, the strange attractor $1 \cdot 2^{x}$ arises. $\lambda_{1}-\lambda_{16}$ are equal to: .00011, .00000, -.00008, $-.00009,-.00011,-.00057,-.00082,-.00136,-.00480$, $-.005700,-.00570,-.00570,-.00937,-.00997,-$ $.01565,-.02348 . \Lambda=-.08329$.

For $G_{0}=16.5$, the strange attractor $1 \cdot 2^{x}$ arises. $\lambda_{1}-\lambda_{16}$ are equal to: .00009, .00000, -.00009, $-.00009,-.00009,-.00057,-.00082,-.00126,-.00475$, $-.00560,-.00560,-.00560,-.00903,-.00997,-.01581$, $-.02325 . \Lambda=-.08244$. 

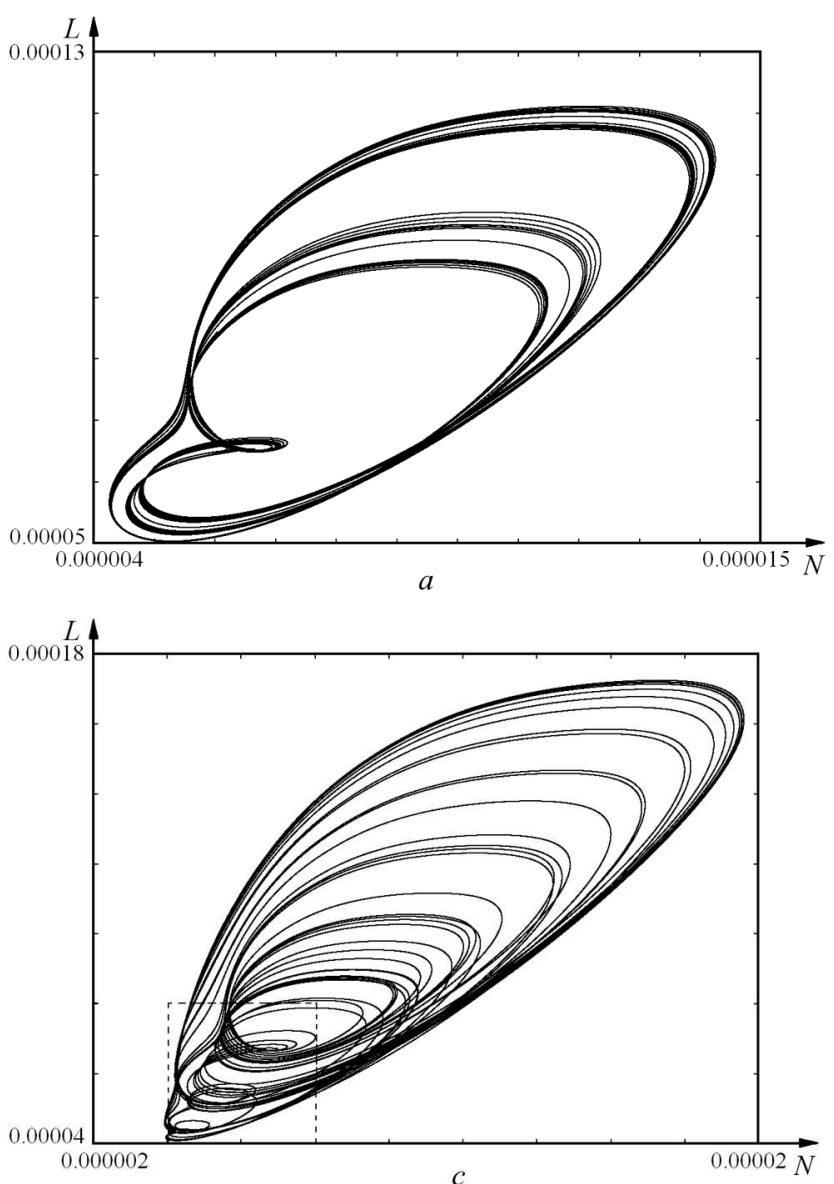
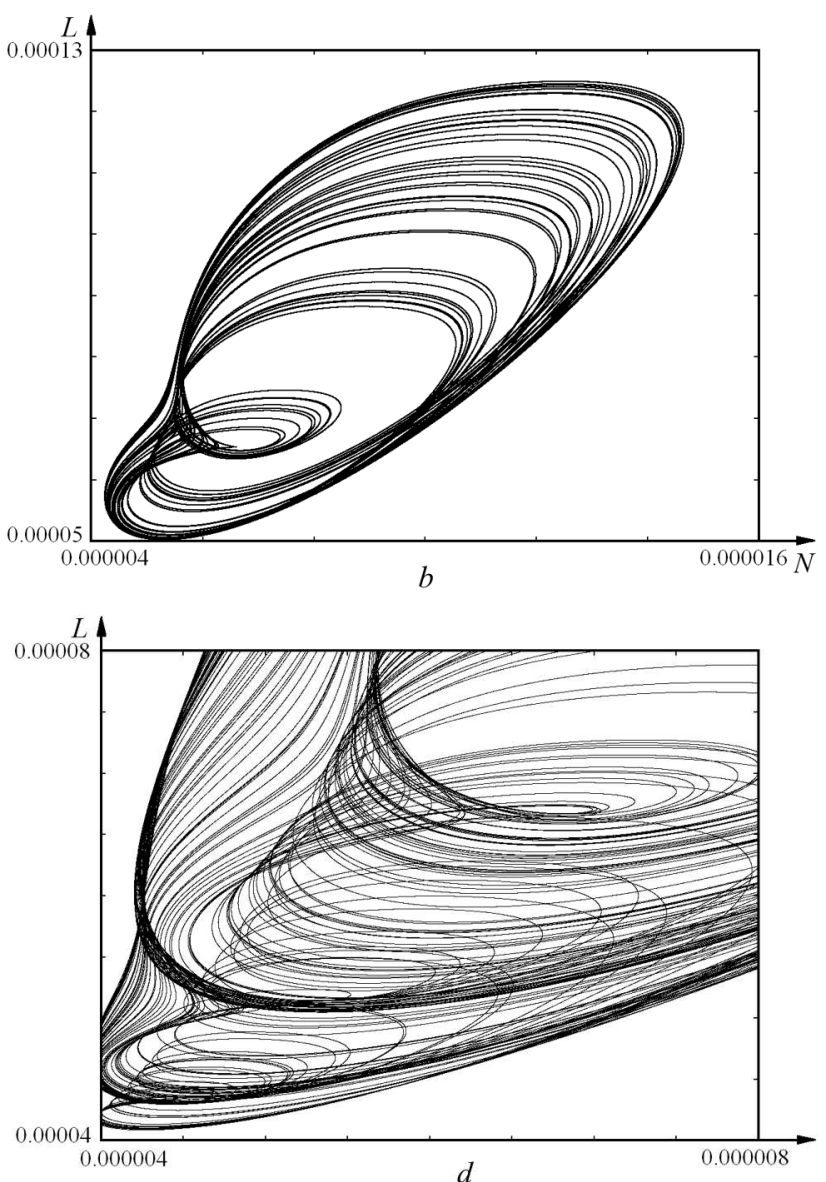

Fig. 5. Projections of the phase portraits of attractors on the plane $(N, L): a-$ regular attractor $1 \cdot 2^{2}$ of a quasistable autoperiodic cycle for $G_{0}=17.25, \lambda_{1}=0 ; b$ - strange attractor $1 \cdot 2^{x}$ for $G_{0}=17.2, \lambda_{1}=0.00004 ; c-$ strange attractor $1 \cdot 2^{x}$ for $G_{0}=16.8, \lambda_{1}=.00011 ; d$ - section of the formation of a deterministic chaos in the mixing funnel (see Fig. $5, c$ )

For $G_{0}=16.3$, the strange attractor $1 \cdot 2^{x}$ arises. $\lambda_{1}-\lambda_{16}$ are equal to: .00004, .00000, -.00008, $-.00009,-.00011,-.00055,-.00081,-.00108,-.00462$, $-.00505,-.00505,-.00505,-.00917,-.00996,-.01676$, $-.02213 . \Lambda=-.08047$.

In Fig. $5, a-d$, we show the projections of the phase portraits of attractors in the plane $(N, L)$, which are formed for the following values of $G_{0}$ : 17.25, 17.2, and 16.8. The larger the positive senior Lyapunov in$\operatorname{dex} \lambda_{1}$, the more unstable is the system (compare Fig. 5, $a-c)$.

In Fig. 5, $c$, we separate a small rectangular section, which cover the funnel of mixing of the given strange attractor, and magnify it (Fig. 5, d). Let us calculate the trajectory on a longer time interval. As is seen, the character of the construction of trajectories of the given strange attractor is repeated on small and large scales of the projection of a phase portrait. Every arising curve of the projection of a chaotic attractor is a source of the formation of new curves. Moreover, the geometric regularity of the construction of trajectories in the phase space is repeated. The given geometric structure reminds a two-scale parametric Cantor set.

By using the Lyapunov indices for strange attractors, we determined the KS-entropy (entropy by Kolmogorov-Sinai) [42]. According to the Pesin theorem [43], the KS-entropy $h$ corresponds to the sum of all positive Lyapunov characteristic indices.

The KS-entropy allows one to judge about the rate of loss of the information about the initial state of the system. The positivity of the entropy is a criterion of 
it. This gives possibility to qualitatively estimate the property of local stability of the attractor.

We determined also the quantity reciprocal to the $\mathrm{KS}$-entropy, $t_{\mathrm{min}}$. It is the time of mixing in the system, which indicates how rapidly the initial conditions will be forgotten. For $t \ll t_{\text {min }}$, the behavior of the system can be predicted with sufficient accuracy. For $t>t_{\min }$, only its probabilistic description is possible. The chaotic mode is unpredictable due to the loss of the memory of initial conditions. The value of $t_{\text {min }}$ is called the Lyapunov index and characterizes the "horizon of predictability" of a strange attractor.

To classify the geometric structure of strange attractors, we calculated the value of their fractality. The strange attractors are fractal sets and have a fractional Hausdorff-Besicovitch dimension. But its direct calculation is a very laborious problem, which has no standard algorithm. Therefore, as the quantitative measure of fractality, we calculated the Lyapunov dimension of attractors by the Kaplan-Yorke formula $[44,45]$ :

$D_{F_{r}}=m+\frac{\sum_{i=1}^{m} \lambda_{i}}{\left|\lambda_{m+1}\right|}$,

where $m$ is the number of the first Lyapunov indices ordered by diminution, whose sum is $\sum_{i=1}^{m} \lambda_{i} \geqslant 0$; $m+1$ is the number of the first Lyapunov index, whose value $\lambda_{m+1}<0$.

For the above-presented strange attractors $2^{x}$, we calculated the following parameters.

For $G_{0}=17.2: h=0.00004, t_{\min }=25000, D_{F_{r}}=$ $=2.667$.

For $G_{0}=16.8: h=0.00011, t_{\min }=9090.9, D_{F_{r}}=$ $=3.375$.

For $G_{0}=16.5: h=0.00009, t_{\min }=11111.1$, $D_{F_{r}}=3.000$.

For $G_{0}=16.3: h=0.00004, t_{\min }=25000, D_{F_{r}}=$ 2.500 .

These results allow us to judge about the difference of the geometric structures of the given strange attractors. The largest value of KS-entropy is obtained for $G_{0}=16.8$. This indicates the highest chaotic mixing of trajectories in a funnel. Respectively, the mixing time, after which the unpredictable chaos arises, will be minimal. The Lyapunov dimension for the given attractor, which characterizes quantitatively the fractality, is maximal in this case. This is confirmed by Fig. 5, $c, d$. By calculating successively the different strange attractors, we can determine a certain regularity in the hierarchy of their chaotic behavior. The geometric shape of attractors of the system varies correspondingly to the change of these indices. Thus, glycolysis in a cell is adapted at the variation of the amount of external glucose $\left(G_{0}\right)$ to the varying conditions of the external environment, by preserving its functionality in this case.

\section{Conclusions}

With the help of the mathematical model, we have studied the metabolic process of glycolysis arising as a product of the biochemical evolution in protobionts. It is shown that glycolysis can be distinguished as a united self-regulating complex of the metabolic network of a cell. The conditions of its selforganization in the cyclic mode are determined. The bifurcations of doubling of a cycle according to the Feigenbaum scenario are discovered, and it is found that the intermittence results finally in the appearance of aperiodic modes of strange attractors. This means that the intensity of the metabolic process of glycolysis is adapted to the varying conditions of the medium. The fractal nature of the obtained cascade of bifurcations is demonstrated. The strange attractors, which are formed due to the formation of a mixing funnel, are found. The complete spectra of Lyapunov indices and the divergences for various modes are calculated. For the strange attractors, the KS-entropies, "horizons of predictability," and the Lyapunov dimensions of attractors are determined. The obtained results allow one to study the structural-functional connections of the metabolic process of glycolysis and their influence on the cyclicity of metabolic oscillations in a cell, as well as to clarify the physical laws of the self-organization in it.

The work is supported by the project No.0112U000056 of the National Academy of Scienses of Ukraine.

1. A.P. Rudenko, DAN SSSR 159, 1374 (1964).

2. A.P. Rudenko, The Theory of Self-Development of Open Catalytic Systems (Moscow State Univ., Moscow, 1969) (in Russian).

3. A.P. Rudenko, Zh. Fiz. Khim. 57, 1597, 2641 (1983); 61, 1457 (1987).

4. G. Nicolis and I. Prigogine, Self-Organization in Nonequilibrium Systems. From Dissipative Structures to Order through Fluctuations (Wiley, New York, 1977). 
5. B. Hess and A. Boiteux, Annu. Rev. Biochem. 40, 237 (1971).

6. J. Higgins, Proc. Nat. Acad. Sci. USA, 51, 989 (1964).

7. E.E. Selkov, Europ. J. Biochem. 4, 79 (1968).

8. A. Goldbeter and R. Lefever, Biophys J. 12, 1302 (1972).

9. L.N. Drozdov-Tikhomirov, G.I. Skurida, and A.A. Alexandrov, J. Biomol. Struct. Dyn. 16, 917 (1999).

10. A. Godlbeter and R. Caplan, Annu. Rev. Biophys. Bioeng. 5, 449 (1976).

11. A.D. Suprun, Yu.I. Prylutskyy, A.M. Shut, and M.S. Miroshnichenko, Ukr. J. Phys. 48, 704 (2003).

12. Yu.I. Prylutskyy, A.M. Shut, M.S. Miroshnychenko, and A.D. Suprun, Int. J. Thermophys. 26, 827 (2005).

13. A.D. Suprun, A.M. Shut, Yu.I. Prylutskyy, Ukr. J. Phys. 52, 997 (2007).

14. Chaos in Chemical and Biochemical System, edit. by R. Field, L. Györgyi (World Scientific, Singapore, 1993).

15. 15. V.P. Gachok and Ya.M. Yakymiv, Dokl. Akad. Nauk SSSR 300, 1095 (1988).

16. V.P. Gachok, Kinetics of Biochemical Processes (Naukova Dumka, Kiev, 1988) (in Russian)

17. V.P. Gachok, Strange Attractors in Biosystems (Naukova Dumka, Kiev, 1989) (in Russian).

18. V.S. Anishchenko, Complex Oscillations in Simple Systems (Nauka, Moscow, 1990) (in Russian).

19. S.P. Kuznetsov, Dynamical Chaos (Fiz.-Mat. Nauka, Moscow, 2001) (in Russian).

20. V.P. Gachok and V.I. Grytsay, Dokl. Akad. Nauk SSSR 282, 51 (1985).

21. V.P. Gachok, V.I. Grytsay, A.Yu. Arinbasarova, A.G. Medentsev, K.A. Koshcheyenko, and V.K. Akimenko, Biotechn. Bioengin 33, 661 (1989).

22. V.P. Gachok, V.I. Grytsay, A.Yu. Arinbasarova, A.G. Medentsev, K.A. Koshcheyenko, and V.K. Akimenko, Biotechn. Bioengin 33, 668 (1989).

23. V.I. Grytsay, Dopov. Nats. Akad. Nauk Ukr., No. 2, 175 (2000).

24. V.I. Grytsay, Dopov. Nats. Akad. Nauk Ukr., No. 3, 201 (2000).

25. V.I. Grytsay, Dopov. Nats. Akad. Nauk Ukr., No. 11, 112 (2000).

26. V.I. Grytsay, Ukr. J. Phys. 46, 124 (2001).

27. V.V. Andreev and V.I. Grytsay, Matem. Modelir. 17, No. 2, 57 (2005).

28. V.V. Andreev and V.I. Grytsay, Matem. Modelir. 17, No. 6, 3 (2005).

29. V.I. Grytsay and V.V. Andreev, Matem. Modelir. 18, No. 12, 88 (2006).

30. V.I. Grytsay, Medium Romanian J. Biophys. 17, No. 1, 55 (2007).

31. V.I. Grytsay, Biofiz. Visn., No. 2, 92 (2007).
32. V.I. Grytsay, Biofiz. Visn., No. 2, 25 (2008).

33. V.I. Grytsay, Ukr. J. Phys. 55, 599 (2010).

34. V.I. Grytsay and I.V. Musatenko, Ukr. Biochem. J. 85, No. 2, 93 (2013).

35. V.I. Grytsay and I.V. Musatenko, Ukr. J. Phys. 58, 677 (2013).

36. V.I. Grytsay and I.V. Musatenko, Chaotic Modeling and Simulation (CMSIM) No. 4, 539 (2013).

37. V.I. Grytsay and I.V. Musatenko, Ukr. Biokhim. Zh. 85, No. 5, 191 (2013).

38. V.I. Grytsay and I.V. Musatenko, Biopol. and Cell 30, 404 (2014).

39. V.I. Grytsay and I.V. Musatenko, Chaotic Modeling and Simulation (CMSIM) 3, 207 (2014).

40. V. Grytsay, Ukr. J. Phys. 60, 564 (2015).

41. M.J. Feigenbaum, J. Stat. Phys. 19, 25 (1978).

42. A.N. Kolmogorov, DAN SSSR 154, 754 (1959).

43. Ya.B. Pesin, Usp. Mat. Nauk. 32, No. 4, 55 (1977).

44. J.L. Kaplan and J.A. Yorke, Ann. N. Y. Acad. Sci. 316, 400 (1979).

45. J.L. Kaplan and J.A. Yorke, in: Functional Differential Equations of Fixed Points, eds. H.O. Peitgen, H.O. Walther (Springer, Berlin, 1979), p. 204.

Received 04.08.15

\section{В.Й. Грицай}

\section{САМООРГАНІЗАЦІЯ І ФРАКТАЛЬНІСТЬ} В МЕТАБОЛІЧНОМУ ПРОЦЕСІ ГЛІКОЛІЗУ

$\mathrm{P}$ е $з$ ю м е

У роботі за допомогою математичної моделі досліджується метаболічний процес гліколізу. Загальна схема гліколізу розглядається як закономірний результат біохімічної еволюції. Використовуючи теорію дисипативних структур, проведено пошук умов самоорганізації даного процесу. Знайдено автокаталітичні процеси, завдяки яким зберігається циклічність в динаміці його протікання. Досліджено умови порушення синхронізації процесу, збільшення кратності циклічності та виникнення хаотичних режимів. Отримано фазопараметричні діаграми каскаду біфуркацій, які відзеркалюють перехід до хаотичних режимів відповідно сценарію Фейгенбаума та перемежаємості. Знайдено дивні аттрактори, що виникають внаслідок воронки. Побудовані їх аттрактори. Розраховані повні спектри показників Ляпунова і дивергенцій для знайдених режимів. Розраховані КС-ентропії, горизонти передбачуваності та ляпуновскі розмірності дивних аттракторів. Зроблено висновки про структурно-функціональні зв'язки гліколізу та їх впливу на стійкість метаболічного процесу клітини. 